\title{
An Elliptical Routing Protocol for Wireless Mesh Networks: Performance Analysis
}

\author{
S Siva Nageswara Rao \\ Research Scholar \\ JNTUK, \\ Kakinada
}

\author{
Y.K.Sundara Krishna, \\ Ph.D \\ Professor \& Principal \\ Krishna University \\ Machilipatnam
}

\author{
K.Nageswara Rao, Ph.D \\ Professor \& Principal \\ PSCMRCET \\ Vijayawada
}

\begin{abstract}
Wireless Mesh Networks (WMN) is one of the implemented types of MANETs. WMN is wireless network, in which each node can communicate directly with one or other peer nodes. In generally, MANET routing protocols are used in WMN, it arises performance issues. In this paper, we propose a new routing protocol called, Elliptical routing protocol, which evaluates the performance via simulator. We show that elliptical routing protocol accomplishes than MANET routing protocols in terms of various routing metrics such as packet delivery ratio, packet loss ratio, routing overhead, throughput, hop count, ETX and ETT. We are also proposing two new routing metrics CLF and RSF, these metrics also shows that, the proposed protocol outperforms the MANET routing protocols.
\end{abstract}

\section{INTRODUCTION}

Wireless Mesh Networking (WMN) [10] is Wireless Local Area Network with certain specialized characteristics such as instant deploy ability, self-configuring, last-mile broadband access provisioning, and low-cost backhaul services for large coverage. A WMN is typically composed of mesh access points, gateways, and wireless clients. The wireless clients mesh access points, and gateways communicate with each other via wireless medium, and form a wireless backhaul in a mesh form. The clients gain network access through mesh access points.

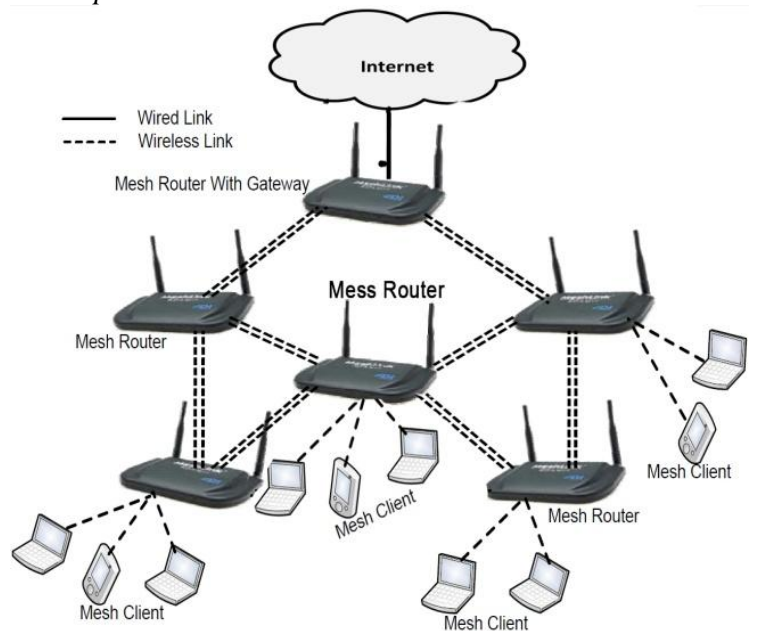

Figure1: Wireless Mesh Networks

It is essential to study the existing routing protocols used in mesh networks and to require developing more efficiency protocol in order to establish efficient routing mechanism in WMN. The performance of protocol can be analyzed using proper metrics. The metrics that are required to analyze the mesh routing protocols are Hop Count, Throughput, Packet delivery ratio, Packet Loss Ratio, Routing overhead Expected Transmission Count (ETX), The Expected transmission time (ETT)[1].

The existing routing algorithms in particular DSR, DSDV and AODV protocols not utilizing bandwidth effectively and also generates unnecessary packets which are sent to different directions. In this paper a new routing approach called elliptical routing protocol is proposed for proper routing of the packets in appropriate directions towards the destination duly saving bandwidth with resulting of better performance compare to existing routing protocols. In this paper we are also proposing two new metrics; they are Communication Load Factor (CLF) and Resource Saving Factor (RSF).

The rest of the paper organized as follows: Section 2 describes related work. Section 3 proposes an elliptical routing protocol for wireless mesh networks. Section 4 presents' protocol implementation and simulation parameters. Section 5 describes performance evaluation and scenarios which are used to investigate the performance of the elliptical protocol. Section 6 concludes the paper.

\section{RELATED WORK}

WMN protocols are either proactive or reactive or hybrid in nature [9].

\subsection{Reactive Routing protocols:}

In a reactive routing protocol, routing paths are searched only when needed. A route discovery operation invokes a routedetermination procedure. The discovery procedure terminates either when a route has been found or no route available after examination for all route permutations. In mobile networks active routes may be disconnected due to node mobility. In WMNs node mobility is very minimal, so reactive routing protocols have better scalability than proactive routing protocols. The reactive routing protocols are Dynamic Source Routing (DSR) protocol, Adhoc On Demand Distance Vector (AODV) protocol, Link Quality Source Routing Algorithm (LQSR) protocol and SrcRR

\subsubsection{Dynamic Source Routing Algorithm (DSR)}

[3] is a unicast reactive routing protocol. It deploys source routing, which means each data packet contains complete routing information. DSR uses flooding.

The DSR protocol has two phases: route discovery phase and route maintenance phase. The first phase is route discovery phase; it is initiated by source node. Source node broadcast the data packets with header. Header includes source address, destination address and unique sequence number. These packets are called Route Request (RREQ) packets.

In the Second phase, when source node wants to send the data, first check the route cache. If the route cache 
available, source node put all the address of nodes for the path to destination in the header .In DSR, when link disconnection is identified during transmission, a route error (RERR) packet is generated and sent to back to the source. When RERR packet reaches to source, again route discovery process is initiated.

The advantages are, Nodes can store multiple paths to destination and it does not require periodic exchanges of Hello messages

The disadvantages are DSR is not suitable for large networks.

\subsubsection{Adhoc on Demand Distance Vector (AODV):}

[6] AODV routing protocol doesn't deploy flooding. AODV is pure on demand route acquisition system, as nodes that are not on a selected path do not maintain routing information or participate in routing table exchanges. AODV stores next hop routing information of the active route at each node.

In AODV, source node wishes to send data packets to destination node; if no path is available, it initiates node discovery process. In AODV, hello messages are used to notify adjacent neighbor nodes. In node discovery process, source node broadcasts route request (RREQ) packets. RREQ packet contains source nodes address, destination nodes address and broadcast id, which is an identifier, and it includes most recent sequence number of destination and source node sequence number.

The advantages are, it does not need any centralized system to handle routing process and AODV is loop free and avoids count to Infinity Problem

The disadvantages are Shortest path may be lost due to traffic during route discover process, Power consumption is also high and It does not avoid Congestion Control

\subsubsection{Link Quality Source Routing Algorithm}

(LQSR)

Link Quality Source Routing Algorithm proposed by Microsoft Research Group. It is a Reactive routing algorithm. It is based on DSR algorithm by improving link quality metrics and other related metrics. To improve the link quality, and LQSR uses link cache instead of route cache.

In LQSR, when a node receives a route request (RREQ) packet, it will adds link quality metric for the link over which packet had arrived. When a Source node receives route reply (RREP) packet, it includes link quality information and node information. For link state information, LQSR sends hello messages to adjacent nodes. These messages are used to measure the link quality at each node for the link on which this message was received. All these messages are based on piggybacked approach.

\subsection{Proactive Routing protocols:}

In proactive routing protocols, mobile nodes continuously evaluate routes to all reachable nodes and attempt to maintain consistent, up-to-date routing information. Therefore, a source node can get a routing path immediately if it needs one. In proactive routing protocols, all nodes need to maintain a consistent view of the network topology. When a network topology change occurs, respective updates must be propagated throughout the network to notify the change. The presence of a fixed wireless backbone seems to favor proactive routing protocols. The proactive routing protocols are Destination-Sequenced Distance-Vector Routing (DSDV), Optimized Link State Routing Protocol (OLSR), Wireless Mesh Networks routing protocol (MRP) [11] and Scalable Routing using heat Protocols.

\subsubsection{Destination Sequenced Distance Vector}

Routing Protocol is proactive unicast routing Protocol. DSDV is based on traditional Bellman Ford algorithm.

In DSDV, every node maintains a routing table .Each entry in a routing table having all possible destinations in the network and number of hops to each destination. Sequence numbers are used in DSDV to avoid loops. The routing updates are either time driven or event driven. Every node periodically transmits routing table updates including its routing information to its adjacent neighbor nodes.

The advantages are, DSDV is an efficient protocol for Route Discovery and DSDV also guarantees Loop -Free Paths

The disadvantages are, when network size, mobility increases delay rate increases, packet delivery ratio decreases and Congestion Control is worst

2.2.2 Link Quality Source Routing Algorithm proposed by Microsoft Research Group. It is a Reactive routing algorithm. It is based on DSR algorithm by improving link quality metrics and other related metrics.

In LQSR, when a node receives a route request (RREQ) packet, it will adds link quality metric for the link over which packet had arrived. When a Source node receives route reply (RREP) packet, it includes link quality information and node information.

The advantage is, It Broadcasts Link State Information rather than routing tables

The disadvantages are Bandwidth is wasted due to periodic updating of nodes information, all control messages should be protected which consumes additional Bandwidth and Scalability

\subsubsection{Scalable Routing using heat Protocol}

Scalable Routing using heat Protocol [9] is Proactive routing protocol. It doesn't deploy flooding. The basic of Heat is to provide Scalability and robustness. Scalability can be achieved by exchanging local messages .Robustness is achieved by assigning the temperature values such that paths through network areas with high redundancy preferred.

In this protocol, gateways are built up as heat resources, which develop the temperature in the network. A node, which has higher temperature, it is nearer to access point, that node performs packet forwarding. So every node measures its own temperature by only evaluating the temperature of its neighbors. Whenever an entry is added, removed or changed, the temperature is re calculated.

\section{AN ELLIPTICAL ROUTING PROTOCOL}

In the mobile adhoc routing environment, node can transmits R_REQ to its entire neighbor's in all directions as shown in figure 2. This type of route finding approach leads to unnecessary overhead due to sending of $R$ _REQ packets in all directions unnecessarily instead of sending in right direction. 


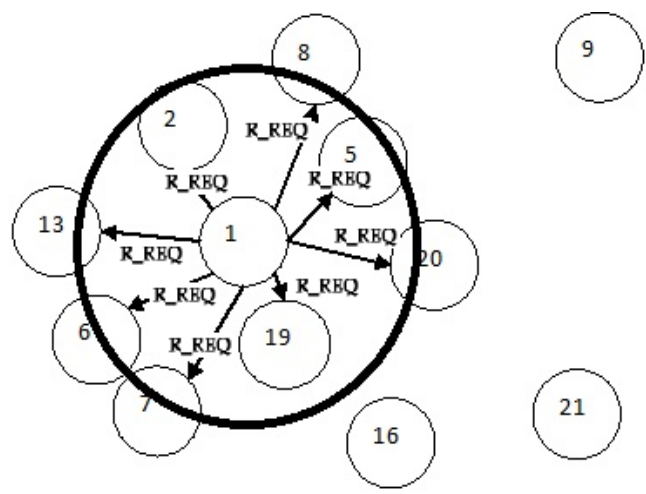

Figure2: Adhoc route establishment approach

In this paper, an elliptical routing approach for sending R_REQ packets is addressed in order to minimize the overhead of R_REQ packets by sending these packets in a direction that is targeting to the required destination by assuming in ellipse towards the destination by selecting the next hop appropriately as shown in figure 3 .

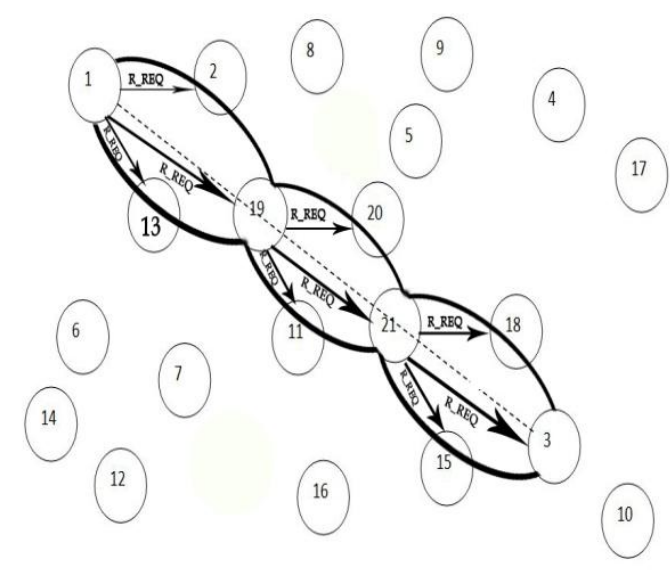

Figure3: Elliptical route establishment approach

\subsection{Description of Elliptical approach.}

The elliptical approach is designed as an algorithm in order to implement the concept in a simulated environment

i Let $\mathbf{S}\left(\mathrm{x}_{\mathrm{s}}, \mathrm{y}_{\mathrm{s}}\right)$ be the position of source node and $\mathbf{D}$ $\left(\mathrm{x}_{\mathrm{d}}, \mathrm{y}_{\mathrm{d}}\right)$ be the position of the Destination node

ii Assume Virtual line between Source $\mathbf{S}$ and Destination D

iii Identify /Specify the first node from Source $\mathrm{S}$ on virtual line, that node can be assumed as Destination $\mathrm{D}_{1}$.

iv Select a node $D_{1}$ among the identified nodes in step III which is either on assumed line or very nearer to assumed line.

$\mathrm{v}$ Let us consider the node selected in step IV as Source node $S_{1}$ and assume virtual line $S_{1}$ to $D$.

vi Repeat Step III to Step V until destination D

vii Identify /Specify the first node from Source $S$ on virtual line, that node can be assumed as Destination D1

viii The distance between Source S and Destination D1 can be taken as major axis [a] and 3/4 or (0.75) of the major axis treated as minor axis [b].Then construct ellipse.

ix The Equation of Ellipse is $\left(\mathrm{x}^{2} / \mathrm{a}^{2}\right)+\left(\mathrm{y}^{2} / \mathrm{b}^{2}\right)=1$ $\mathrm{x}$ In the next step, D1 can be assumed as S1 and identify the next node, which can be nearer in the line or on the line, that node can be treated as D2. The distance between Source S1 and Destination D2 can be taken as major axis [a] and $3 / 4$ or $(0.75)$ of the major axis treated as minor axis [b].Then construct the Ellipse.

xi This procedure is followed until all ellipses are constructed towards Destination D or Dn.

xii Send data from source $S$ to destination $D$ through assumed nodes those on line

xiii If any nodes moves follow the above steps

\subsection{Algorithm}

Definitions:

Sal: Source altitude

Sla: Source latitude

Slo: Source longitude

Dal: Destination altitude

Dla: Destination latitude

Dlo: Destination longitude

Vline: Virtual line

[Assume that minor axis (b) can be taken as $3 / 4$ of the major axis (a)]

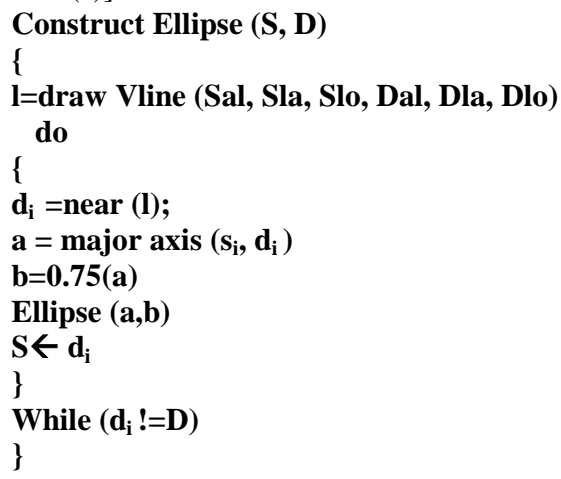

\section{IMPLEMENTATION OF PROTOCOL AND PERFORMANCE EVALUATION 4.1 Protocol Implementation}

We modify the source code of AODV in NS-2 (v2.35) to implement the proposed protocol. The proposed elliptical protocol transmits the Hello packets to obtain the neighbor nodes information, and also needs to carry the neighbor list in the R_REQ packet in a unidirectional way. Therefore, in implementation, elliptical approach is used to reduce the overhead of Hello packets and neighbor list in the R_REQ packet, which are described as follows:

In order to reduce the overhead of Hello packets, we do not use periodical Hello mechanism. Since a node sending any packets can inform its neighbors of its existence, the broadcasting packets such as R_REQ and route error (R_ERR) can play a role of Hello packets.

\subsection{Simulation Environment}

In order to evaluate the performance of the proposed elliptical protocol, we compare it with some other protocols using the NS-2 simulator. In order to compare the routing performance of the proposed protocol, we choose the elliptical Route protocol which is an optimization scheme for reducing the overhead of R_REQ packet incurred in route discovery and also provides the scalability.

Simulation parameters are as follows: The radio channel having a bit rate of $3 \mathrm{Mbps}$, and the transmission range is 250 meters. We consider constant bit rate (CBR) [7] data traffic 
and every source sends CBR packets whose size is 1040 bytes per second.

Table: 1 Simulation Parameters

\begin{tabular}{|lc|}
\hline Simulation Parameter & \multicolumn{1}{c|}{ Value } \\
\hline $\begin{array}{l}\text { Simulator } \\
(2.35)\end{array}$ & \\
\hline Topology $2500 \mathrm{~m} * 2500 \mathrm{~m}$ \\
\hline $\begin{array}{l}\text { No of Nodes } \\
10,20,50,100,200,500,1000\end{array}$ \\
\hline Transmission Range & $250 \mathrm{~m}$ \\
\hline Bandwidth & $3 \mathrm{Mbps}$ \\
\hline Queue Length & 50 \\
\hline Packet Size & $1040 \mathrm{bytes}$ \\
\hline Pause Time & $0 \mathrm{~s}$ \\
\hline Min Speed & $1 \mathrm{~m} / \mathrm{s}$ \\
\hline Max Speed & $10-100 \mathrm{~m} / \mathrm{s}$ \\
\hline Simulation Time & $120 \mathrm{~s}$ \\
\hline We evaluate the performance of routing protocols using
\end{tabular}

We evaluate the performance of routing protocols using the following metrics

Packet Delivery Ratio: Packet Delivery Ratio (PDR) is defined as the total no of packets successfully received by the destination to the no of packets sent by the source.

PDR= Number of Packets Received $(\mathrm{ACK}) /$ Number of Packets Sent (TCP).

The PDR specifies the performance of protocol that how successfully the packets have been transferred

Packet Loss Ratio: Packet Loss Ratio (PLR) is defined as the difference between the no of packets sent by the source and received by the sink

Routing Overhead :Routing overhead is defined as the ratio of total no of routing packets to the data packets which has been calculated at the MAC layer.

Throughput: Throughput is defined as the total no of bits delivered by total duration of simulation time.

Hop Count: It is defined as the number of hops between the source and destination of a path. It ignores issues such as link load and link quality

Expected Transmission Count: Expected Transmission Count (ETX) is defined as the number of transmissions required to successfully deliver a packet over a wireless link. The ETX path metric is simply the sum of the ETX values of the individual links. ETX is a measure of link and path quality.

The ETX metric for a single link is defined as shown below, where $d f$ is the measured rate or probability that a packet will be successfully delivered in the forward direction and $d r$ denotes the probability that the corresponding acknowledgement packet is successfully received.

\section{ETX $=1 /($ df $\times d r)$}

Expected Transmission Time: The Expected transmission time (ETT) metric is an extension of ETX which considers different link routes or capacities. ETT is simply the expected time to successfully transmit a packet at the MAC layer and is defined as follows for a single link:

\section{$\mathbf{E T T}=\mathbf{E T X} \times(\mathbf{S} / \mathbf{B})$}

$S$ denotes the average size of a packet and $B$ the current link bandwidth. The ETT path metric is obtained by adding up all the ETT values of the individual links in the path. Low ETT values are desired.

Communication Load Factor: The Communication Load Factor (CLF) is defined as the total no of nodes involved in communication in elliptical approach by total no of nodes are participated in communication in adhoc approach.

$$
\mathbf{C L F}=\mathbf{N}_{\mathrm{e}} / \mathbf{N}_{\mathbf{a}}
$$

In MANET, more no of nodes are involved in route discovery process. Where as in elliptical protocol can significantly reduce the CLF incurred during route discovery process it can proceed unidirectional only, so only few no of nodes can participated. The advantage of CLF is to provide the scalability.

Resource Saving Factor: The Resource Saving Factor (RSF) is the differences between the resources used in adhoc approach and elliptical approach for route establishment by the resources used in adhoc approach for route establishment.

\section{$\mathbf{R S F}=\left(\mathbf{R} \mathbf{R E Q N}_{\mathrm{a}}-\mathbf{R} \mathrm{REQN}_{\mathrm{e}}\right) / \mathbf{R} \mathbf{R E Q N}_{\mathrm{a}}$}

In MANET, R_REQ can be transmitted in al directions. So it can consume lot bandwidth for route discovery process. In elliptical routing protocol, $\mathrm{R} \_\mathrm{REQ}$ can be transmitted only one direction, it can save bandwidth

This advantage of RSF specifies how much bandwidth saves when we are using elliptical routing protocol compared to adhoc routing protocols.

\section{PERFORMANCE ANALYSIS}

Figure 4 shows the packet delivery ratio with increasing no of nodes. The Elliptical routing protocol can increase the packet delivery ratio because it reduces the no of collisions as shown in figure 4 , so that it reduces the number of packet drops caused by collisions. When network size is up to 20 nodes, the packet delivery ratio of elliptical protocol marginally better than AODV, DSR and DSDV protocols. When network size increases up to 1000 nodes, the elliptical protocol increases the packet delivery ratio about 7 and 5 percent when compared with DSR, DSDV and AODV protocols respectively.

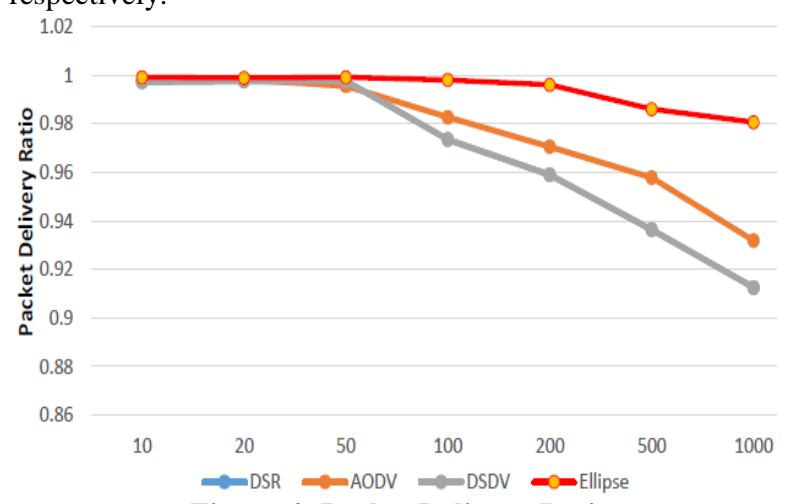

Figure 4: Packet Delivery Ratio 


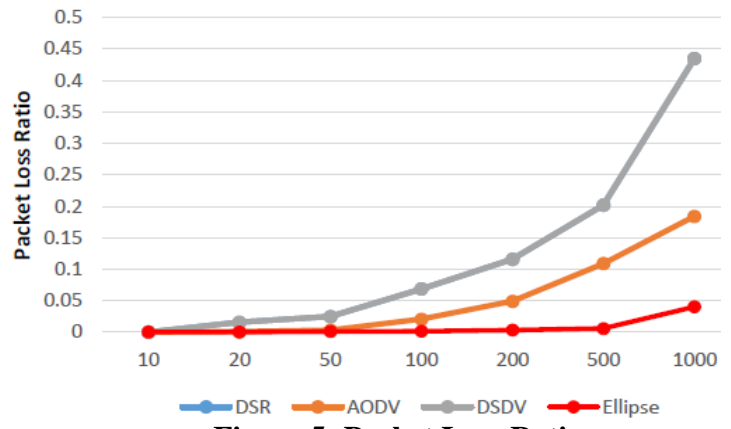

Figure 5: Packet Loss Ratio

In the IEEE 802.11 protocol, the data and control packets can be sent on the same channel. In the conventional routing protocols, massive redundant rebroadcasting occurs due to interference [7] and collisions, which leads to excessive dropping. This packet dropping not only affects the number of retransmissions but also affects the packet delivery ratio. This phenomenon will be more severe with an increase in the number no of nodes.

Figure 5 shows the packet loss ratio with increasing no of nodes. The Elliptical routing protocol can decreases the packet loss ratio because it reduces the no of collisions as shown in figure 5. When network size is up to 20 nodes, the packet loss ratio of elliptical protocol marginally better than AODV, DSR and DSDV protocols. When network size increases up to 1000 nodes, the elliptical protocol decreases the packet loss ratio about 37 and 14 percent when compared with DSR, DSDV and AODV protocols respectively.

Figure 6 shows the routing overhead with different number of nodes. The elliptical protocol can significantly reduce the routing overhead incurred during route discovery process. Although the elliptical routing protocol increases the packet size of RREQ, it significantly reduces more no of RREQ packets. When the network size is up to 50 nodes, routing overhead of elliptical protocol marginally better than AODV, DSR and DSDV protocols. When network size up to 1000 nodes, elliptical protocol reduces routing overhead about 22 and 42 percent when compared to DSR DSDV and AODV protocols.

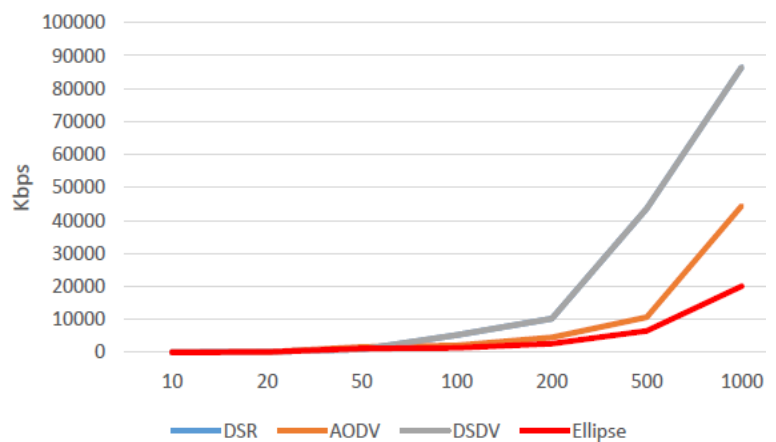

Figure 6: Routing overhead

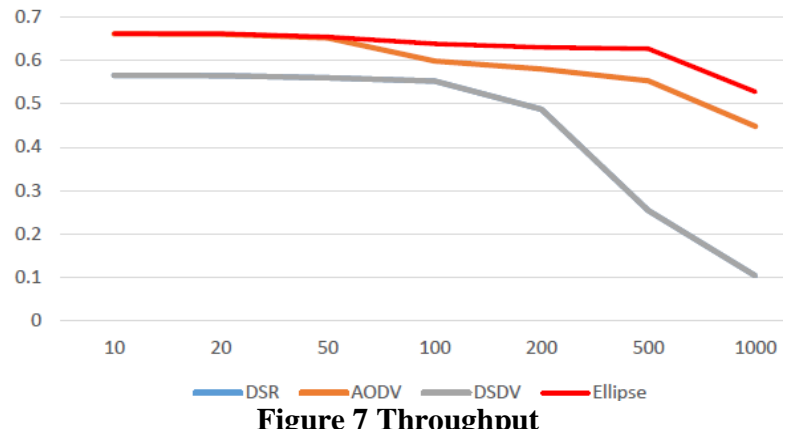

Figure 7 Throughput

Figure 7 shows the throughput with increasing no of nodes. The Elliptical routing protocol can increase the throughput because it reduces the no of collisions and packet drops caused by collisions. When network size is up to 50 nodes, throughput of elliptical protocol marginally better than AODV, DSR and DSDV protocols. When network size increases up to 1000 nodes, the elliptical protocol increases the throughput about 42 and 7 percent when compared with DSR, DSDV and AODV protocols respectively.

Hop Count with increased nodes is showed in figure 8. When the network size is up to 50 to 1000 nodes, hop count of elliptical protocol similar and marginally better than AODV, DSR and DSDV.

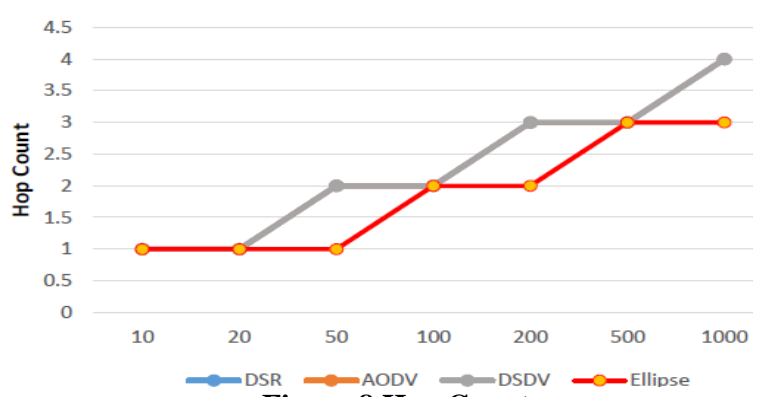

Figure 8 Hop Count

Figure 9 shows the ETX with increasing no of nodes. The Elliptical routing protocol can decrease the ETX because it having efficient link quality and path as shown in figure 9. When network size is up to 50 nodes, ETX of elliptical protocol marginally better than AODV, DSR and DSDV protocols. When network size increases up to 1000 nodes, the elliptical protocol reduces the ETX when compared with DSR, AODV and DSDV protocols respectively.

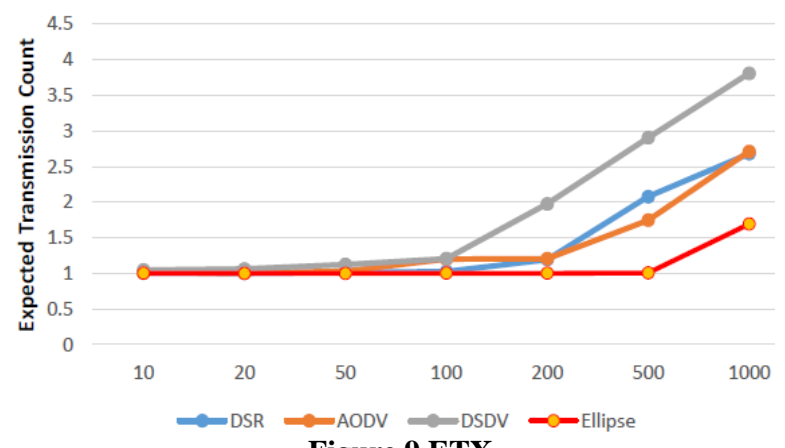

Figure 9 ETX 


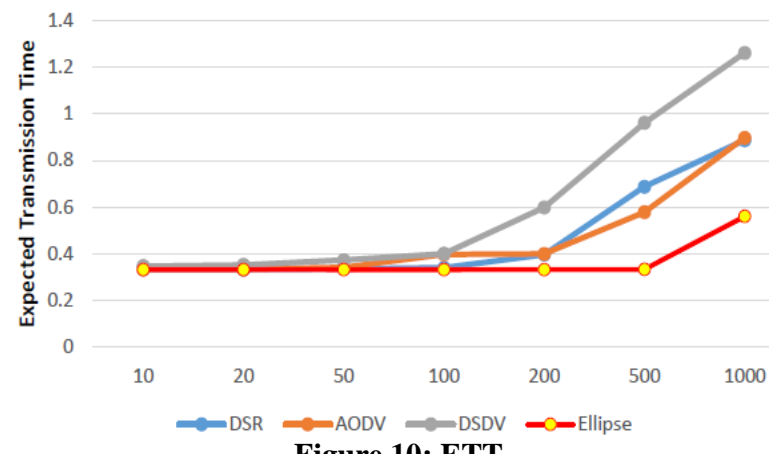

Figure 10: ETT

Figure 10 shows the ETT with increasing no of nodes. The Elliptical routing protocol can decrease the ETT because it increases the link capacities as shown in figure 10 . When network size is up to 50 nodes, ETT of elliptical protocol marginally better than AODV, DSR and DSDV protocols. When network size increases up to 1000 nodes, the elliptical protocol reduces the ETT when compared with DSR, AODV and DSDV protocols respectively.

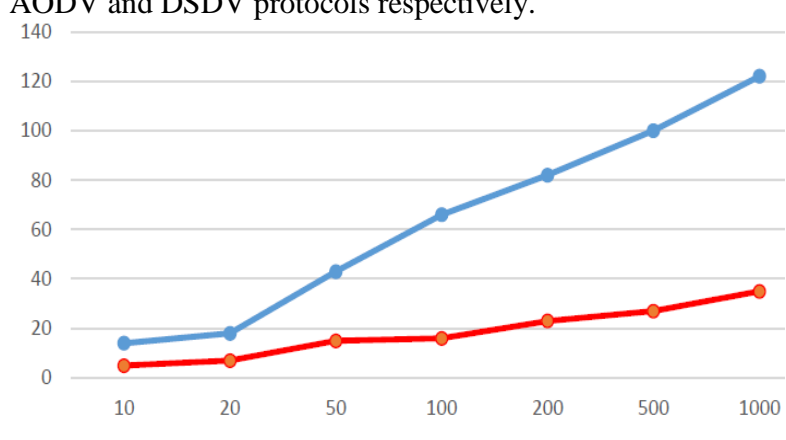

Figure 11: CLF

Figure 11 shows the CLF with different number of nodes. The elliptical protocol can significantly reduce the CLF incurred during route discovery process it can proceed unidirectional only, so only few no of nodes can participated. When the network size is up to 50 nodes, CLF of elliptical protocol marginally better than AODV, DSR and DSDV protocols. When network size up to 1000 nodes, elliptical protocol reduces CLF when compared to DSR DSDV and AODV protocols.

Figure 12 shows the RSF with different number of nodes. The elliptical protocol can significantly reduce the RSF incurred during route discovery process in can proceed unidirectional only, so only few no of R_REQ can be transmitted for route discovery process.so it consumes less bandwidth when compared to MANET routing protocols. When the network size is up to 50 nodes, RSF of elliptical protocol marginally better than AODV, DSR and DSDV protocols. When network size up to 1000 nodes, elliptical protocol reduces RSF when compared to DSR DSDV and AODV protocols.

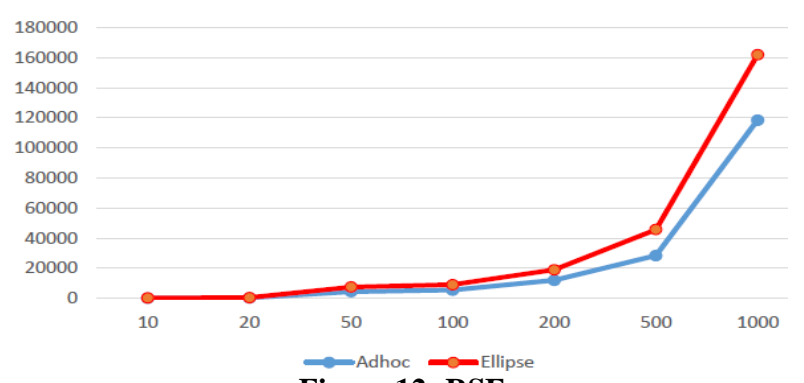

Figure 12: RSF

\section{CONCLUSION}

Wireless Mesh Networks are turn into very popular because they have significant advantages over other networks. In this paper, we proposed a new elliptical protocol, which is specially designed for wireless mesh networks. The design of elliptical protocol takes benefits of particularities of mesh networks only maintain the routes and data forwarding can be done with in the ellipses only. Simulation results show that, elliptical protocol efficiently outperforms the existing MANET routing protocols.

\section{REFERENCES}

[1] Y. Yang, J. Wang, and R. Kravets, "Designing Routing Metrics forMesh Networks," IEEE Workshop on Wireless Mesh Networks (WiMesh),Proceedings of the, June 2005.

[2] Sonia Waharte, Raouf Boutaba, Youssef Iraqi, Brent Ishibashi, Routing protocols in wireless mesh networks: challenges and design considerations

[3] J. Broch, D. B. Johnson, D. A. Maltz, The Dynamic Source Routing Protocol for Mobile Ad Hoc Networks," IETF Internet Draft draft-ietf-manet-dsr-01.txt, December 1998

[4] Rainer Baumann, Simon Heimlicher, Mario Strasser, Andreas Weibel, "A Survey on Routing Metrics". February 10, 2007

[5] S.S.Tyagi and R.K.Chauhan "Performance Analysis of proactive and reactive routing protocols for Adhoc Networks".

[6] C. E. Perkins and E. M. Royer, Ad-hoc On-Demand Distance Vector Routing," Proceedings of 2nd IEEE Workshop on Mobile Computing Systems and Applications, February 1999.

[7]Zhang et AL: A Neighbor coverage based probabilistic rebroadcasr for reducing routing overhead in Mobile Adhoc Networks: IEEE transactions on Mobile Computing

[8]SSN Rao, YKS Krishna, K.N Rao, "Performance Evaluation of routing protocols in Wireless Mesh networks ",International journal of Computer applications(0975-8887) vol68- April 2013.

[9] S. Siva Nageswara Rao, Y. K. Sundara Krishna, and K.Nageswara Rao,"A Survey: Routing Protocols for Wireless Mesh Networks”, IJRRWSN Vol. 1, No. 3.

[10] F. Akyildiz, X. Wang, and W. Wang, "Wireless mesh networks: a survey," Computer Networks, 2005.

[11] Jangeun Jun, Mihail L. Sichitiu, MRP: Wireless mesh networks routing protocol, Computer Networks Journal (2008). 